\title{
Activation of a HERV-H LTR induces expression of an aberrant calbindin protein in human prostate carcinoma cells Eva Gebefügi ${ }^{1}$, Reinhard Brunmeir ${ }^{2}$, Claudia Weierich ${ }^{3}$, Horst Wolff ${ }^{1}$, Ruth Brack-Werner ${ }^{1}$ and Christine Leib-Mösch*1,4
}

Address: ${ }^{1}$ Institute of Virology, Helmholtz Zentrum München, German Research Center for Environmental Health, Neuherberg, Germany, ${ }^{2}$ Institute of Medical Biochemistry, Medical University of Vienna, Max Perutz Laboratories, Vienna, Austria, ${ }^{3}$ Department of Biology II, LMU Munich, Martinsried, Germany and ${ }^{4}$ Medical Clinic III, Medical Faculty Mannheim, University of Heidelberg, Germany

* Corresponding author

from Frontiers of Retrovirology: Complex retroviruses, retroelements and their hosts Montpellier, France. 2I-23 September 2009

Published: 24 September 2009

Retrovirology 2009, 6(Suppl 2):P48 doi:10.1 186/1742-4690-6-S2-P48

This abstract is available from: http://www.retrovirology.com/content/6/S2/P48

(c) 2009 Gebefügi et al; licensee BioMed Central Ltd.

The human genome contains around 500000 LTR retrotransposons including endogenous retroviruses (HERVs). Most of these LTR elements are silenced by epigenetic conditions, but may be reactivated by environmental factors such as chemicals, radiation or exogenous viruses. We have analyzed a HERV-H element located upstream of the human calbindin gene (CALB-1) that leads to expression of a truncated calbindin protein by alternative splicing in a human prostate carcinoma cell line (PC3). PC3 cells are polyploid with one or two complete alleles of chromosome 8 , and four to five pieces of the 8q-arm, all containing the CALB-1 locus. Analysis of these loci did not reveal substantial alterations on DNA sequence level, such as modification of splice sites, suggesting an epigenetic activation of the HERV-H LTR. Therefore, we compared the DNA methylation status of this LTR in PC3 cells and in three prostate carcinoma cell lines not expressing the truncated calbindin protein. We found that the HERV-H LTR is hypomethylated in two cell lines including PC3. Chromatin immunoprecipitation (ChIP) analysis, however, revealed that the chromatin is associated with active marks (acetylated histone $\mathrm{H} 4$ and tri-methylated lysine4 at histone H3) at this locus only in PC3 cells. This data suggests that reactivation of the HERV-H LTR requires at least two steps: demethylation of DNA and modification of histones. There is evidence that expression of the truncated calbindin prevents apoptosis and may thus contribute to the malignant phenotype of PC3 cells. 